\title{
The Use of KSpaceNavigator as a Tool for Defect Analysis by Diffraction Contrast Microscopy
}

\author{
A. Gautam T. Duden and U. Dahmen \\ National Center for Electron Microscopy, Materials Sciences Division, LBNL, Berkeley, CA 94720
}

For quantitative characterization of defects in materials, diffraction contrast imaging remains one of the most important tools in electron microscopy. Experiments such as a $\mathrm{g} \cdot \mathrm{b}$ analysis to determine the Burgers vector of a dislocation, the displacement vector of a stacking fault or the strain field of an inclusion are key to the analysis of materials and their structural defects. Typically diffraction contrast experiments require a series of images of the same defect under a series of orientations given by the need to view a crystal from a series of well-defined angles, sometimes requiring a precision of milliradians. These requirements place great demands on operator skill and patience. Frequently, a series of experiments must be abandoned when the area of interest is lost from the field of view during sample tilting, or when the orientation needed is just beyond the tilt range of the stage. To assist with experiments that require extensive and precise tilting in order to view the same nanoparticle or defect under different diffracting conditions, it is highly desirable to have tool that helps translate between stage and crystal coordinates. The KSpaceNavigator software was developed to fill this need [1]. Initially designed as a support tool for the TEAM stage, the software is applicable to any double-tilt or tilt-rotation stage on any microscope. It can be used to execute experiments, minimize the dose to the sample, or plan experiments to ensure they are feasible within the available tilt range of a given stage. These features can be of great practical use for many diffraction contrast experiments. This talk illustrates their use in the analysis of multiply-twinned precipitates in a polycrystalline matrix.

The morphology of Ge precipitates in an Al matrix has been shown to be dominated by the tendency of the precipitates to form twins. A detailed analysis showed that twinning is directly responsible for precipitate shapes. Untwinned particles form octahedra, single twins lead to triangular plates, multiple twins on the same plane cause hexagonal plates, while twinning on non-parallel twin systems promote tetrahedral precipitates $[2,3]$. To confirm this twinning hypothesis required a series of diffraction contrast experiments to bring each twin separately into diffracting condition. These experiments are particularly complex and time-consuming for tetrahedral particles, which according to the model must have two, three or four separate twins. Detailed confirmation of this hypothesis was constrained by the complexity of the diffraction contrast experiments. Here we demonstrate an application of KSpaceNavigator to this experiment, using a Philips CM200 microscope with a double-tilt side-entry stage and a tilt range of about $\pm 25^{\circ}$.

Diffraction patterns were recorded directly from the fluorescent screen using an external point-andshoot digital camera. Employing a simple calibration procedure included in the software, these patterns were used to set up the transformation from stage coordinates to crystal coordinates. Once established, this transformation could be used to determine the precise alpha and beta tilt coordinates needed to tilt the sample into the required orientations. The great advantage of this procedure is the ability to tilt the sample in real space while keeping the area of interest in the field of view, without the need for frequent checks of the diffraction pattern to ascertain and correct the orientation. This virtually eliminates the danger of losing the area of interest during an experiment and greatly reduces 
the sample exposures to the beam because it makes it possible to reach target orientations quickly and in a single step. This capability is equally useful for discrete zone axis tomography, or for 3D imaging under weak beam conditions.

In this presentation, the utility of the software will be demonstrated with a range of experiments of interest to practicing microscopists, and its adaptation to different microscopes and stages and varying degrees of automation will be outlined [4].

\section{References}

[1] T. Duden et al., Microsc. Microanal. 15(Suppl 2) (2009) 1228.

[2] S.Q. Xiao et al., Philosophical Magazine A 73 (1996) 1261.

[3] S. Hinderberger et al., Z. Metallk. 91 (2000) 215

[4] This work is supported by the Director, Office of Science, Office of Basic Energy Sciences, of the U.S. Department of Energy under Contract No. DE-AC02-05CH11231.
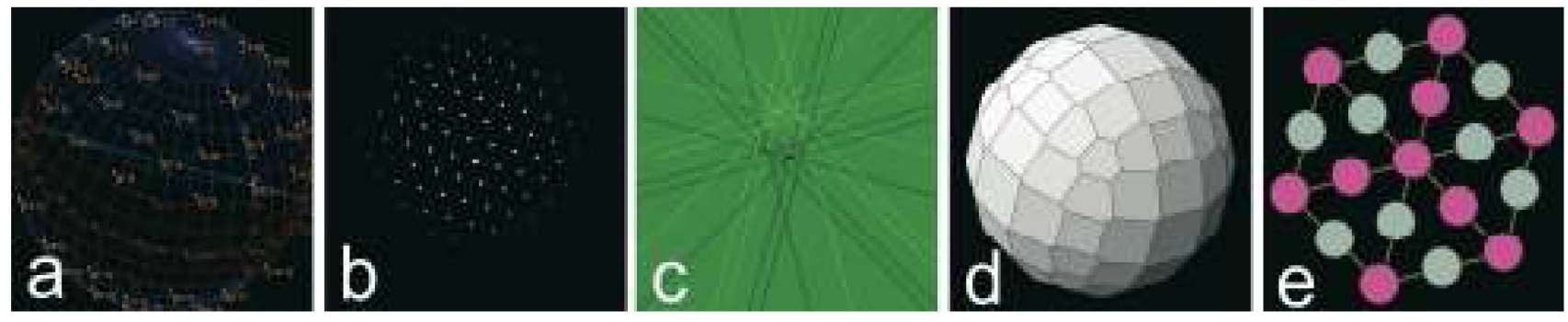

FIG. 1. User interface for KSpaceNavigator showing tools available to assist the operator with experimental planning, calibration, crystal tilting, and analysis of diffraction patterns and crystal shapes; (a) control sphere, (b) kinematic diffraction pattern, (c) kinematic Kikuchi line pattern, (d) Wulff shape plot, (e) projection of unit cell.
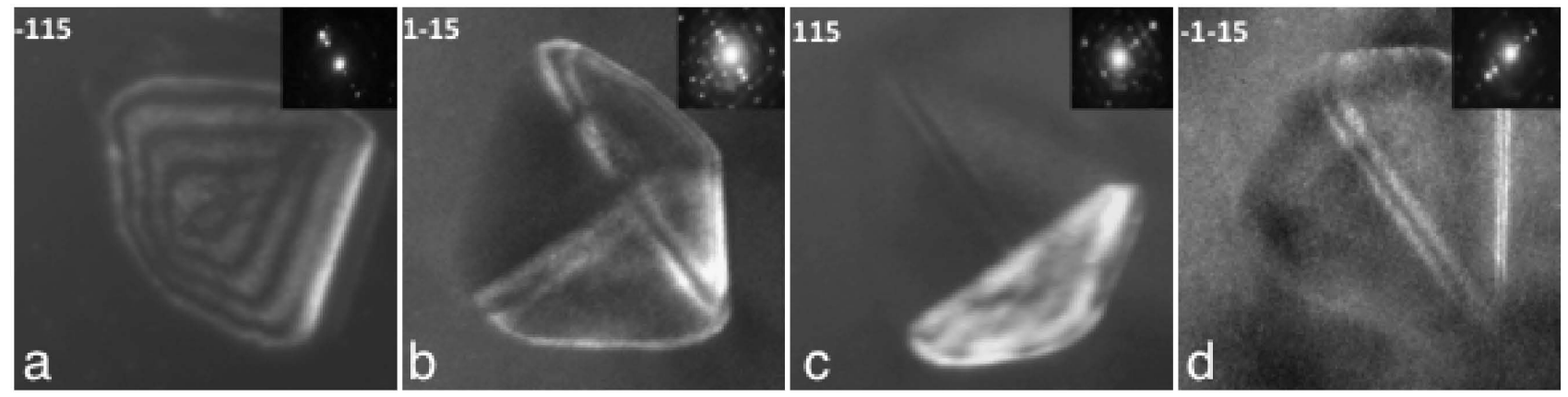

FIG. 2. Tetrahedral Ge precipitate imaged in a sequence of $g_{220}$ dark field images to show 3D shape and twin structure of the particle. The initial sample orientation near a [001] zone axis was used to calibrate the stage to the sample coordinates. To test for twinning, the sample was tilted to all four neighboring $<115>$ orientations, each of which is parallel to a $<111>$ orientation of one of the four possible primary twins. Only two of the four twins are present in this precipitate $(b, c)$. 